\title{
NEW EXACT ANALYTICAL SOLUTIONS FOR THE GENERAL KDV EQUATION WITH VARIABLE COEFFICIENTS
}

\author{
Bao-Jian Hong ${ }^{1,2}$ and Dian-Chen $\mathrm{Lu}^{1, *}$ \\ ${ }^{1}$ Faculty of Science, Jiangsu University, 212013, Zhenjiang, Jiangsu, PR China \\ ${ }^{2}$ Department of Basic Courses, Nanjing Institute of Technology, 211167,Nanjing, \\ Jiangsu, PR China \\ ludianchenujs@163.com,dclu@ujs.edu.cn
}

\begin{abstract}
In this paper, a general algebraic method based on the generalized Jacobi elliptic functions expansion method, the improved general mapping deformation method and the extended auxiliary function method with computerized symbolic computation is proposed to construct more new exact solutions of a generalized $\mathrm{KdV}$ equation with variable coefficients. As a result, eight families of new generalized Jacobi elliptic function wave solutions and Weierstrass elliptic function solutions of the equation are obtained by using this method, some of these solutions are degenerated to soliton-like solutions, trigonometric function solutions in the limit cases when the modulus of the Jacobi elliptic functions $m \rightarrow 1$ or 0 , which shows that the general method is more powerful and will be used in further works to establish more entirely new solutions for other kinds of nonlinear partial differential equations arising in mathematical physics.
\end{abstract}

Key words- Generalized KdV equation with variable coefficients; general algebraic method; exact solutions; generalized Jacobi elliptic function wave-like solutions

\section{INTRODUCTION}

Nonlinear partial differential equations (NLPDEs) are widely used to describe complex physical phenomena arising in the world around us and various fields of science. The investigation of exact solutions of NLPDEs plays an important role in the study of these phenomena such as the nonlinear dynamics and the mechanism behind the phenomena. With the development of soliton theory, many powerful methods for obtaining exact solutions of NLPDEs have been presented, such as inverse scattering transformation [1], Hirota bilinear method [2], Bäcklund transformation [3], Darboux transformation [4], homotopy perturbation method [5], extended Riccati equation rational expansion method [6], asymptotic methods [7], extended auxiliary function method [8], algebraic method [9], Jacobi elliptic function expansion method [10], and so on [11-13].

In [14][15], Hong proposed a generalized Jacobi elliptic functions expansion method to obtain generalized exact solutions of NLPDEs. In [16], Hong et al. proposed an 
improved general mapping deformation method to obtain generalized exact solutions of the general $\mathrm{KdV}$ equation with variable coefficients (GVKDV). Which is more general than many other algebra expansion methods [6,8-15] etc. The solution procedure of this method, by the help of Matlab or Mathematica, is of the utmost simplicity, and this method can be easily extended to all kinds of NLPDEs.

In this work, we will proposed the general algebraic method to obtain several new families of exact solutions for the GVKDV equations.

The rest of this paper is organized as follows. In section 2, we briey describe the new general algebraic method. In section 3, several families of solutions for the GVKdV equation are obtained, some of which are degenerated to new solitary-like solutions and new triangular-like functions solutions in the limit case. In section 4, some conclusions are given.

\section{SUMMARY OF THE GENERAL ALGEBRAIC METHOD}

Consider a given nonlinear evolution equation with one physical field $u(x, t)$ in two variables $x$ and $t$

$$
P\left(u, u_{t}, u_{x}, u_{x x}, \cdots\right)=0 \text {. }
$$

We seek the following formal solutions of the given system by a new intermediate transformation:

$u(\xi)=\sum_{i=0}^{n} A_{i}(t) \varphi^{i}(\xi)+\sum_{i=-n}^{-1} A_{n-i}(t) \varphi^{i}(\xi)$.

Where $A_{i}(t), A_{n-i}(t)$ are functions of $t$ to be determined later. $\xi=\xi(x, t)$ are arbitrary functions with the variables $x$ and $t$. The parameter $n$ can be determined by balancing the highest order derivative terms with the nonlinear terms in Eq.(1). And $\varphi(\xi)$ is a solution of the following ordinary differential equation (ODE)

$$
\varphi^{\prime 2}(\xi)=\sum_{i=0}^{4} a_{i}(t) \varphi^{i}(\xi)
$$

Substituting Eqs. (3) and (2) into Eq. (1), and setting the coefficients of $\varphi^{i}(\xi)(i=0,1,2, \cdots)$ and $x^{s} \varphi^{j}(\xi) \sqrt{\sum_{i=0}^{4} a_{i}(t) \varphi^{i}(\xi)}(s=0,1 ; j=\cdots,-2,-1,0,1,2, \cdots)$ to zero yield a set of algebraic equations for $A_{i}(t), A_{n-i}(t)$ and $\xi$. Using the Mathematica to solve the algebraic 
equations and substituting each of the solutions of the set, i.e. each of the expressions of $\varphi(\xi)$ into Eq. (2), we can get the solutions of Eq. (1). In order to obtain some new general solutions of Eq.(3),we assume that (3) have the following solutions:

$\varphi(\xi)=c_{0}+c_{1} e(\xi)+c_{2} f(\xi)+c_{3} g(\xi)+c_{4} h(\xi)$.

Where $c_{i}=c_{i}(t)(i=0, \cdots, 4)$ are functions of $t$ to be determined later, the four functions $e=e(\xi), f=f(\xi), g=g(\xi), h=h(\xi)$ are expressed as the follows:

$e=\frac{1}{p+q F+r F^{2}+l F^{\prime}}, f=\frac{F}{p+q F+r F^{2}+l F^{\prime}}, g=\frac{F^{2}}{p+q F+r F^{2}+l F^{\prime}}, h=\frac{F^{\prime}}{p+q F+r F^{2}+l F^{\prime}}$.

Where $p, q, r, l$ are arbitrary constants which ensure denominator unequal to zero, so do the following situations, and $F=F(\xi)$ is a solution of the following ODE $F^{\prime 2}=A+B F^{2}+C F^{4}+2 D F+2 E F^{3}, \quad F^{\prime \prime}=B F+2 C F^{3}+D+3 E F^{2}$. Where "'” denotes $\frac{d}{d \xi}$, “"” denotes $\frac{d^{2}}{d \xi^{2}}, A, B, C, D, E$ are arbitrary constants, so do the following situations, the four functions $e, f, g, h$ satisfy the following relations:

$$
\left\{\begin{array}{l}
e^{\prime}=-q e h-2 r f h-l\left(D e^{2}+B e f+2 C f g+3 E f^{2}\right), \\
f^{\prime}=p e h-r g h+l\left(A e^{2}+D e f-C g^{2}-E f g\right), \\
g^{\prime}=q g h+2 p f h+l\left(2 A e f+3 D f^{2}+B f g+E g^{2}\right), \\
h^{\prime}=(D p-A q) e^{2}+(B p-D q-2 A r) e f+(2 C p+E q-B r) f g+3(E p-D r) f^{2}+(C q-E r) g^{2} \\
f^{2}=e g, h^{2}=A e^{2}+B f^{2}+C g^{2}+2 D e f+2 E f g, p e+q f+r g+l h=1
\end{array}\right.
$$

And $e, f, g, h$ satisfy one of the following relations at the same time.

Family 1:When $p=0$

$$
\left(C l^{2}-r^{2}\right) h^{2}=-C+2 C l h-B r(1-l h-q f) e-A e^{2} r^{2}-2 D r^{2} e f+(2 C q-2 E r) f+(2 l E r-2 C l q) f h+\left(2 E q r-C q^{2}\right) f^{2} .(7 \mathrm{a})
$$

Family 2:When $q=0$ 
$\left(C l^{2}-r^{2}\right) h^{2}=2 C(l h+p e-p l e h)+2 E r(l h-1) f-B r(1-l h-p e) e-\left(C p^{2}+A r^{2}\right) e^{2}+\left(2 E p r-2 D r^{2}\right) e f-C$.

Family 3: When $r=0$

$C l^{2} g^{2}=1-2 E l^{2} f g-2 p e+\left(p^{2}-A l^{2}\right) e^{2}-2 q f+2\left(p q-D l^{2}\right) e f+\left(q^{2}-B l^{2}\right) e g$.

Family 4 :When $l=0$

$r^{2} h^{2}=C-2 C p e+(2 E r-2 C q) f+\left(C p^{2}+A r^{2}\right) e^{2}+\left(C q^{2}-2 E q r+B r^{2}\right) e g+\left(2 C p q-2 E p r+2 D r^{2}\right) e f$.

Substituting (4),(5),(6),(7) along with (7a)-(7d) into Eq.(3) separately yields four families of polynomial equations for $e, f, g, h$.Setting the coefficients of $e^{i}, e^{i} f, e^{i} g, e^{i} h, e^{i} f g, e^{i} f h$,

$e^{i} g h(i=0,1,2, \cdots)$ to zero yields a set of over-determined differential equations(ODEs) in

$p, q, r, l, a_{i} 、 c_{i}(i=0,1,2,3,4), A, B, C, D, E \quad$ and $\quad \xi(x, t)$,solving the ODEs by Mathematica and $\mathrm{Wu}$ elimination, we can obtain many exact solutions of Eq.(1) accroding to (2),(3),(4),(5),(6).

If we let $c_{0}=c_{1}=c_{3}=c_{4}=0, c_{2}=1, p=1, q=r=l=0, a_{0}=A, a_{1}=2 D, a_{2}=B, a_{3}=2 E$,

$a_{4}=C$, we have $\varphi(\xi)=F(\xi)$, our method contain the improved general mapping deformation method[16]etc.

Remark 1. Our method proposed here is more general than the extended Riccati equation rational expansion method[6], the extended auxiliary function method [8], the generalized F-expansion method[13], the generalized Jacobi elliptic functions expansion method[14,15], and many other algebra expansion methods[9,10,12] [16,17,18,19] etc.

Remark 2. Eq.(2) and Eq.(3) can be extended to the following forms $u(\xi)=\sum_{i=0}^{n} A_{i}(t) \varphi^{i}(\xi)+\sum_{i=-n}^{-1} A_{n-i}(t) \varphi^{i}(\xi)+\sum_{i=-n}^{n} B_{i}(t) \varphi^{i}(\xi) \varphi^{\prime}(\xi), \varphi^{\prime 2}(\xi)=\sum_{i=0}^{4} a_{i}(t) \varphi^{i}(\xi)$.

Where $n$ is usually a positive integer. If $n$ is a fraction or a negative integer, we make the following transformation:

(a) when $n=d / c$ is a fraction, we let $u(\xi)=v^{d / c}(\xi)$, then return to determine the balance constant $n$ again;

(b) when $n$ is a negative integer, we suppose $u(\xi)=v^{n}(\xi)$, then return to determine 
the balance constant $n$ again.

Remark 3. Noticed that

$$
\begin{aligned}
& \left.\left.F_{1}(\xi)\right|_{(A, B, C, D, E)_{1}} \rightarrow \varphi_{1}(\xi)\right|_{\left(a_{0}, a_{1}, a_{2}, a_{3}, a_{4}\right)_{1}}=\left.F_{2}(\xi)\right|_{(A, B, C, D, E)_{2}} \\
& \left.\rightarrow \varphi_{2}(\xi)\right|_{\left(a_{0}, a_{1}, a_{2}, a_{3}, a_{4}\right)_{2}}=\left.F_{3}(\xi)\right|_{(A, B, C, D, E)_{3}} \rightarrow \cdots
\end{aligned}
$$

We find a meanful conclusion that this general method imply a BT of Eq.(1) with the compatible conditions (4),(5),(6),(7) and (7a)-(7d).

In the following, we will use this method to solve the GVKdV equation

\section{EXACT SOLUTIONS TO THE GVKDV EQUATION}

We consider the following GVKdV equation [16-20].

$u_{t}+2 \beta(t) u+[\alpha(t)+\beta(t) x] u_{x}-3 M \gamma(t) u u_{x}+\gamma(t) u_{x x x}=0$

Where $\alpha(t), \beta(t)$ and $\gamma(t)$ are arbitrary functions of $t$. Equation (8) can be reduced to other more physical forms [21-26] which has been discussed in Ref. [16].

By balancing the highest-order linear term $u_{x x x}$ and the nonlinear $u u_{x}$ in (8), we obtain $n=2$, thus we assume that (8) have the following solutions:

$$
\begin{aligned}
& u(\xi)=A_{0}(t)+A_{1}(t) \varphi(\xi)+A_{2}(t) \varphi^{2}(\xi)+A_{3}(t) \varphi^{-1}(\xi)+A_{4}(t) \varphi^{-2}(\xi), \\
& \xi(x, t)=k(t) x+\omega(t) .
\end{aligned}
$$

Where $k(t), \omega(t), A_{i}(t)(i=0,1,2,3,4)$ are functions of $t$ to be determined later.

Substituting (3) (12) and (13) into (8) and setting the coefficients of $\varphi^{i}(\xi)(i=0,1,2, \cdots)$ and $x^{s} \varphi^{j}(\xi) \sqrt{\sum_{i=0}^{4} a_{i}(t) \varphi^{i}(\xi)}(s=0,1 ; j=\cdots,-2,-1,0,1,2, \cdots) \quad$ to $\quad$ zero $\quad$ yield $\quad$ a $\quad$ set $\quad$ of over-determined equations (ODEs) for $A_{i}(t), A_{n-i}(t), k(t), \omega(t)$ and $a_{i}(t)$. After solving the ODEs by Mathematica we could determine the following solutions: 


\section{Family 1}

$$
\begin{aligned}
& a_{2}=\frac{a_{3}^{2}}{4 a_{4}}+\frac{2 a_{1} a_{4}}{a_{3}}, \\
& A_{i}^{\prime}=-2 A_{i} \beta,(i=0,1,2,3,4), k^{\prime}=-k \beta, \\
& A_{1}=2 a_{3} k^{2} / M, A_{2}=4 a_{4} k^{2} / M, A_{3}=0, A_{4}=0, \\
& \omega^{\prime}=k\left[-\alpha+3 M A_{0} \gamma-k^{2} \gamma\left(\frac{8 a_{1} a_{4}}{a_{3}}+\frac{a_{3}^{2}}{4 a_{4}}\right)\right] .
\end{aligned}
$$

\section{Family 2}

$$
\begin{aligned}
& a_{2}=\frac{a_{1}^{2}}{4 a_{0}}+\frac{2 a_{0} a_{3}}{a_{1}}, \\
& A_{i}^{\prime}=-2 A_{i} \beta,(i=0,1,2,3,4), k^{\prime}=-k \beta, \\
& A_{1}=0, A_{2}=0, A_{3}=2 a_{1} k^{2} / M, A_{4}=4 a_{0} k^{2} / M, \\
& \omega^{\prime}=k\left[-\alpha+3 M A_{0} \gamma-k^{2} \gamma\left(\frac{8 a_{0} a_{3}}{a_{1}}+\frac{a_{1}^{2}}{4 a_{0}}\right)\right] .
\end{aligned}
$$

Substituting (4),(5),(6),(7) along with (7a)-(7d) and (11) into Eq.(3) separately yields an ODEs, after solving the ODEs by Mathematica and Wu elimination, we can obtain the following solutions of Eq.(3) and Eq.(8) according to (4),(5),(6) and (12).

\section{Case 1}

$$
\begin{aligned}
& A=1, B=-m^{2}-1, C=m^{2}, D=E=0, F=s n \xi, 0 \leq m \leq 1, \\
& a_{0}=1, a_{1}= \pm 4 \sqrt{2(1+m) \sqrt{m}}, a_{3}=\mp 2 \sqrt{2(1+m) \sqrt{m}}\left(1+6 m+m^{2}-4(1+m) \sqrt{m}\right), \\
& a_{2}=12(1+m) \sqrt{m}-6 m-m^{2}-1, a_{4}=8 m(m+1)^{2}-2(1+m)\left(1+m^{2}+6 m\right) \sqrt{m} \text {, } \\
& p=1, q=\mp \sqrt{2(1+m) \sqrt{m}}, r=m, l=0, c_{0}=c_{1}=c_{3}=c_{4}=0, c_{2}=1, \\
& \varphi_{1}\left(\xi_{1}\right)=\frac{s n \xi_{1}}{1 \mp \sqrt{2(1+m) \sqrt{m}} s n \xi_{1}+m s n^{2} \xi_{1}}, \\
& \xi_{1}=k_{5} e^{-\int \beta(t) d t} x+\int k_{5} e^{-\int \beta(t) d t}\left[-\alpha(t)+\left(3 M k_{0}-k_{5}^{2}\left(36(1+m) \sqrt{m}-6 m-m^{2}-1\right)\right) e^{-2 \int \beta(t) d t} \gamma(t)\right] d t, \\
& u_{1}=k_{0} e^{-2 \int \beta(t) d t} \mp \frac{4 \sqrt{2(1+m) \sqrt{m}}\left(1+6 m+m^{2}-4(1+m) \sqrt{m}\right) k_{5}^{2} e^{-2 \int \beta(t) d t} s n \xi_{1}}{M\left(1 \mp \sqrt{2(1+m) \sqrt{m}} s n \xi_{1}+m s n^{2} \xi_{1}\right)} \\
& +\frac{\left(32 m(m+1)^{2}-8(1+m)\left(1+m^{2}+6 m\right) \sqrt{m}\right) k_{5}^{2} e^{-2 \int \beta(t) d t} s n^{2} \xi_{1}}{M\left(1 \mp \sqrt{2(1+m) \sqrt{m}} s n \xi_{1}+m s n^{2} \xi_{1}\right)^{2}} .
\end{aligned}
$$


Case 2

$A=1, B=-m^{2}-1, C=m^{2}, D=E=0,0 \leq m \leq 1, F=s n \xi$,

$a_{0}=1, a_{1}=\mp 4 \sqrt{1-m^{2}}, a_{2}=8-4 m^{2}, a_{3}=\mp 8 \sqrt{1-m^{2}}, a_{4}=4-4 m^{2}$,

$p=0, q= \pm \sqrt{1-m^{2}}, r=0, l=1, c_{0}=c_{1}=c_{3}=c_{4}=0, c_{2}=1$,

$\varphi_{2}\left(\xi_{2}\right)=\frac{s n \xi_{2}}{ \pm \sqrt{1-m^{2}} s n \xi_{2}+c n \xi_{2} d n \xi_{2}}$,

$\xi_{2}=k_{5} e^{-\int \beta(t) d t} x+\int k_{5} e^{-\int \beta(t) d t}\left[-\alpha(t)+\left(3 M k_{0}-k_{5}^{2}\left(20-16 m^{2}\right)\right) e^{-2 \int \beta(t) d t} \gamma(t)\right] d t$,

$u_{2}=k_{0} e^{-2 \int \beta(t) d t} \mp \frac{16 \sqrt{1-m^{2}} k_{5}^{2} e^{-2 \int \beta(t) d t} s n \xi_{2}}{M\left( \pm \sqrt{1-m^{2}} s n \xi_{2}+c n \xi_{2} d n \xi_{2}\right)}+\frac{16\left(1-m^{2}\right) k_{5}^{2} e^{-2 \int \beta(t) d t} s n^{2} \xi_{2}}{M\left( \pm \sqrt{1-m^{2}} s n \xi_{2}+c n \xi_{2} d n \xi_{2}\right)^{2}}$.

Case 3

$A=1-m^{2}, B=2 m^{2}-1, C=-m^{2}, D=E=0,0 \leq m \leq 1, F=c n \xi$,

$a_{0}=1, a_{1}=-4, a_{2}=8-4 m^{2}, a_{3}=8 m^{2}-8, a_{4}=4-4 m^{2}$,

$p=0, q=1, r=0, l= \pm 1, c_{0}=c_{1}=c_{3}=c_{4}=0, c_{2}=1$,

$\varphi_{3}\left(\xi_{3}\right)=\frac{c n \xi_{3}}{c n \xi_{3} \mp s n \xi_{3} d n \xi_{3}}$,

$\xi_{3}=k_{5} e^{-\int \beta(t) d t} x+\int k_{5} e^{-\int \beta(t) d t}\left[-\alpha(t)+\left(3 M k_{0}-k_{5}^{2}\left(20-4 m^{2}\right)\right) e^{-2 \int \beta(t) d t} \gamma(t)\right] d t$,

$u_{3}=k_{0} e^{-2 \int \beta(t) d t}+\frac{16\left(m^{2}-1\right) k_{5}^{2} e^{-2 \int \beta(t) d t} c n \xi_{3}}{M\left(c n \xi_{3} \mp s n \xi_{3} d n \xi_{3}\right)}+\frac{16\left(1-m^{2}\right) k_{5}^{2} e^{-2 \int \beta(t) d t} c n^{2} \xi_{3}}{M\left(c n \xi_{3} \mp s n \xi_{3} d n \xi_{3}\right)^{2}}$.

Case 4

$$
\begin{aligned}
& A=1-m^{2}, B=2 m^{2}-1, C=-m^{2}, D=E=0,0 \leq m \leq 1, F=c n \xi, \\
& a_{0}=\left(1-c_{0}^{2}\right)\left[1+\left(c_{0}^{2}-1\right) m^{2}\right], a_{1}=2\left(c_{0}-2 c_{0} m^{2}+2 c_{0}^{3} m^{2}\right), a_{2}=2 m^{2}-6 c_{0}^{2} m^{2}-1, \\
& a_{3}=4 c_{0} m^{2}, a_{4}=-m^{2}, p=1, q=0, r=0, l=0, c_{1}=c_{2}=c_{4}=0, c_{3}=1, \\
& \varphi_{4}\left(\xi_{4}\right)=c_{0}+c n \xi_{4}, \\
& \left.\xi_{4}=k_{5} e^{-\int \beta(t) d t} x+\int k_{5} e^{-\int \beta(t) d t}\left[-\alpha(t)+\left(3 M k_{0}-4 k_{5}^{2}\left(2 m^{2}-3 c_{0}^{2} m^{2}-1\right)\right)\right) e^{-2 \int \beta(t) d t} \gamma(t)\right] d t, \\
& u_{4}=\left[k_{0}+\frac{8 c_{0} m^{2} k_{5}^{2}}{M}\left(c_{0}+c n \xi_{4}\right)-\frac{4 m^{2} k_{5}^{2}}{M}\left(c_{0}+c n \xi_{4}\right)^{2}\right] e^{-2 \int \beta(t) d t} .
\end{aligned}
$$

Remark 4: $u_{4}$ are in full agreement with the results in Ref.[16], which contain the results (19) constructed by Zhao in Ref. [17] and $u_{3}$ obtained by Zhu in Ref. [18]. 
Case 5

$$
\begin{aligned}
& A=m^{2}-1, B=2-m^{2}, C=-1, D=E=0,0 \leq m \leq 1, F=d n \xi, \\
& a_{0}=1, a_{1}=-4 m, a_{2}=8 m^{2}-4, a_{3}=8 m-8 m^{3}, a_{4}=4 m^{4}-4 m^{2}, \\
& p=0, q=m, r=0, l= \pm 1, c_{0}=c_{1}=c_{3}=c_{4}=0, c_{2}=1, \\
& \varphi_{5}\left(\xi_{5}\right)=\frac{d n \xi_{5}}{d n \xi_{5} \mp m^{2} s n \xi_{5} c n \xi_{5}}, \\
& \xi_{5}=k_{5} e^{-\int \beta(t) d t} x+\int k_{5} e^{-\int \beta(t) d t}\left[-\alpha(t)+\left(3 M k_{0}-k_{5}^{2}\left(20 m^{2}-4\right)\right) e^{-2 \int \beta(t) d t} \gamma(t)\right] d t, \\
& u_{5}=k_{0} e^{-2 \int \beta(t) d t}+\frac{16 m\left(1-m^{2}\right) k_{5}^{2} e^{-2 \int \beta(t) d t} d n \xi_{5}}{M\left(d n \xi_{5} \mp m^{2} s n \xi_{5} c n \xi_{5}\right)}+\frac{16 m^{2}\left(m^{2}-1\right) k_{5}^{2} e^{-2 \int \beta(t) d t} d n^{2} \xi_{5}}{M\left(d n \xi_{5} \mp m^{2} s n \xi_{5} c n \xi_{5}\right)^{2}} .
\end{aligned}
$$

Case 6

$$
\begin{aligned}
& A=\frac{C_{1} C_{3} q-5 C_{3}^{2} q^{3}+\varepsilon\left(C_{1}+3 C_{3} q^{2}\right) \sqrt{C_{3}\left(3 C_{3} q^{2}-2 C_{1}\right)}}{4 C_{3}}, B=0, C=0,2 D=C_{1}, 2 E=C_{3}, \\
& F=\wp\left(\frac{\sqrt{C_{3}}}{2} \xi, \frac{-4 C_{1}}{C_{3}}, \frac{-4 M}{C_{3}}\right), a_{0}=0, a_{1}=C_{3}, a_{2}=-3 C_{3} q, a_{3}=C_{1}+3 C_{3} q^{2}, \\
& a_{4}=\frac{-3 C_{1} C_{3} q-9 C_{3}^{2} q^{3}+\varepsilon\left(C_{1}+3 C_{3} q^{2}\right) \sqrt{C_{3}\left(3 C_{3} q^{2}-2 C_{1}\right)}}{4 C_{3}}, \varepsilon=\operatorname{sgn}\left[C_{1}+3 C_{3} q^{2}\right], \\
& p=0, q=\mathrm{const}, r=1, l=0, c_{0}=c_{1}=c_{3}=c_{4}=0, c_{2}=1, \\
& \varphi_{6}\left(\xi_{6}\right)=\frac{1}{q+\wp\left(\frac{\sqrt{C_{3}}}{2} \xi_{5}, \frac{-4 C_{1}}{C_{3}}, \frac{-4 M}{C_{3}}\right)}, \\
& \xi_{6}=k_{5} e^{-\int \beta(t) d t} x+\int k_{5} e^{-\int \beta(t) d t}\left[-\alpha(t)+\left(3 M k_{0}-\frac{3}{2}\left(\varepsilon \sqrt{C_{3}\left(3 C_{3} q^{2}-2 C_{1}\right)}-5 C_{3} q\right) k_{5}^{2}\right) e^{-2 \int \beta(t) d t} \gamma(t)\right] d t, \\
& u_{6}=\left[k_{0}+\frac{2\left(C_{1}+3 C_{3} q^{2}\right) k_{5}^{2}}{M} \varphi_{6}\left(\xi_{6}\right)+\frac{\left(C_{1}+3 C_{3} q^{2}\right)\left(\varepsilon \sqrt{C_{3}\left(3 C_{3} q^{2}-2 C_{1}\right)}-3 C_{3} q\right) k_{5}^{2}}{M C_{3}} \varphi_{6}^{2}\left(\xi_{6}\right)\right] e^{-2 \int \beta(t) d t} .
\end{aligned}
$$

Substituting (4),(5),(6),(7) along with (7a)-(7d) and (13) into Eq.(3) separately yields an ODEs, after solving the ODEs by Mathematica and $\mathrm{Wu}$ elimination, we can obtain the following solutions of Eq.(3) and Eq.(8) according to (4),(5),(6) and (14).

Case 7

$$
\begin{aligned}
& A=1, B=-m^{2}-1, C=m^{2}, D=E=0,0 \leq m \leq 1, F=s n \xi, a_{0}=1, a_{1}=-4 q \neq 0, \\
& a_{2}=6 q^{2}-m^{2}-6 m-1, a_{3}=2 q\left(1+6 m+m^{2}-2 q^{2}\right), a_{4}=\left((1+m)^{2}-q^{2}\right)\left(4 m-q^{2}\right), \\
& p=0, q=m, r=0, l= \pm 1, c_{0}=c_{1}=c_{3}=c_{4}=0, c_{2}=1,
\end{aligned}
$$




$$
\begin{aligned}
& \varphi_{7}\left(\xi_{7}\right)=\frac{s n \xi_{7}}{1+q s n \xi_{7}+m s n^{2} \xi_{7}}, \\
& \xi_{7}=k_{5} e^{-\int \beta(t) d t} x+\int k_{5} e^{-\int \beta(t) d t}\left[-\alpha(t)+\left(3 M k_{0}+4 k_{5}^{2}\left(1+6 m+m^{2}-3 q^{2}\right)\right) e^{-2 \int \beta(t) d t} \gamma(t)\right] d t, \\
& u_{7}=\left[k_{0}-\frac{8 q k_{5}^{2}}{M}\left(n s \xi_{7}+q+m s n \xi_{7}\right)+\frac{4 k_{5}^{2}}{M}\left(n s \xi_{7}+q+m s n \xi_{7}\right)^{2}\right] e^{-2 \int \beta(t) d t} .
\end{aligned}
$$

Case 8

$$
\begin{aligned}
& A=1, B=-m^{2}-1, C=m^{2}, D=E=0,0 \leq m \leq 1, F=s n \xi \\
& a_{0}=1, a_{1}= \pm 4 \sqrt{1-m^{2}}, a_{2}=8-4 m^{2}, a_{3}=8 \sqrt{1-m^{2}}, a_{4}=4-4 m^{2}, \\
& p=0, q=\mp \sqrt{1-m^{2}}, r=0, l=1, c_{0}=c_{1}=c_{3}=c_{4}=0, c_{2}=1, \\
& \varphi_{8}\left(\xi_{8}\right)=\frac{s n \xi_{8}}{\mp \sqrt{1-m^{2}} s n \xi_{8}+c n \xi_{8} d n \xi_{8}}, \\
& \xi_{8}=k_{5} e^{-\int \beta(t) d t} x+\int k_{5} e^{-\int \beta(t) d t}\left[-\alpha(t)+\left(3 M k_{0}-k_{5}^{2}\left(20-4 m^{2}\right)\right) e^{-2 \int \beta(t) d t} \gamma(t)\right] d t, \\
& u_{8}=\left[k_{0} \pm \frac{8 \sqrt{1-m^{2}} k_{5}^{2}}{M}\left(c s \xi_{8} d s \xi_{8} \mp \sqrt{1-m^{2}}\right)+\frac{4 k_{5}^{2}}{M}\left(\operatorname{cs} \xi_{8} d s \xi_{8} \mp \sqrt{1-m^{2}}\right)^{2}\right] e^{-2 \int \beta(t) d t} .
\end{aligned}
$$

Case 9

$$
\begin{aligned}
& A=m^{2}-1, B=2-m^{2}, C=-1, D=E=0,0 \leq m \leq 1, F=d n \xi, \\
& a_{0}=1, a_{1}=-4 m, a_{2}=8 m^{2}-4, a_{3}=8 m-8 m^{3}, a_{4}=4 m^{4}-4 m^{2}, \\
& p=0, q=m, r=0, l= \pm 1, c_{0}=c_{1}=c_{3}=c_{4}=0, c_{2}=1, \\
& \varphi_{9}\left(\xi_{9}\right)=\frac{d n \xi_{9}}{d n \xi_{9} \mp m^{2} s n \xi_{9} c n \xi_{9}}, \\
& \xi_{9}=k_{5} e^{-\int \beta(t) d t} x+\int k_{5} e^{-\int \beta(t) d t}\left[-\alpha(t)+\left(3 M k_{0}-k_{5}^{2}\left(20 m^{2}-16\right)\right) e^{-2 \int \beta(t) d t} \gamma(t)\right] d t, \\
& u_{9}=\left[k_{0}-\frac{8 m k_{5}^{2}}{M}\left(1 \mp m^{2} s d \xi_{9} c d \xi_{9}\right)+\frac{4 k_{5}^{2}}{M}\left(1 \mp m^{2} s d \xi_{9} c d \xi_{9}\right)^{2}\right] e^{-2 \int \beta(t) d t} .
\end{aligned}
$$

We can give the numerical simulation of $u_{2}$ and $u_{7}$ (see Figs. 1-2). 


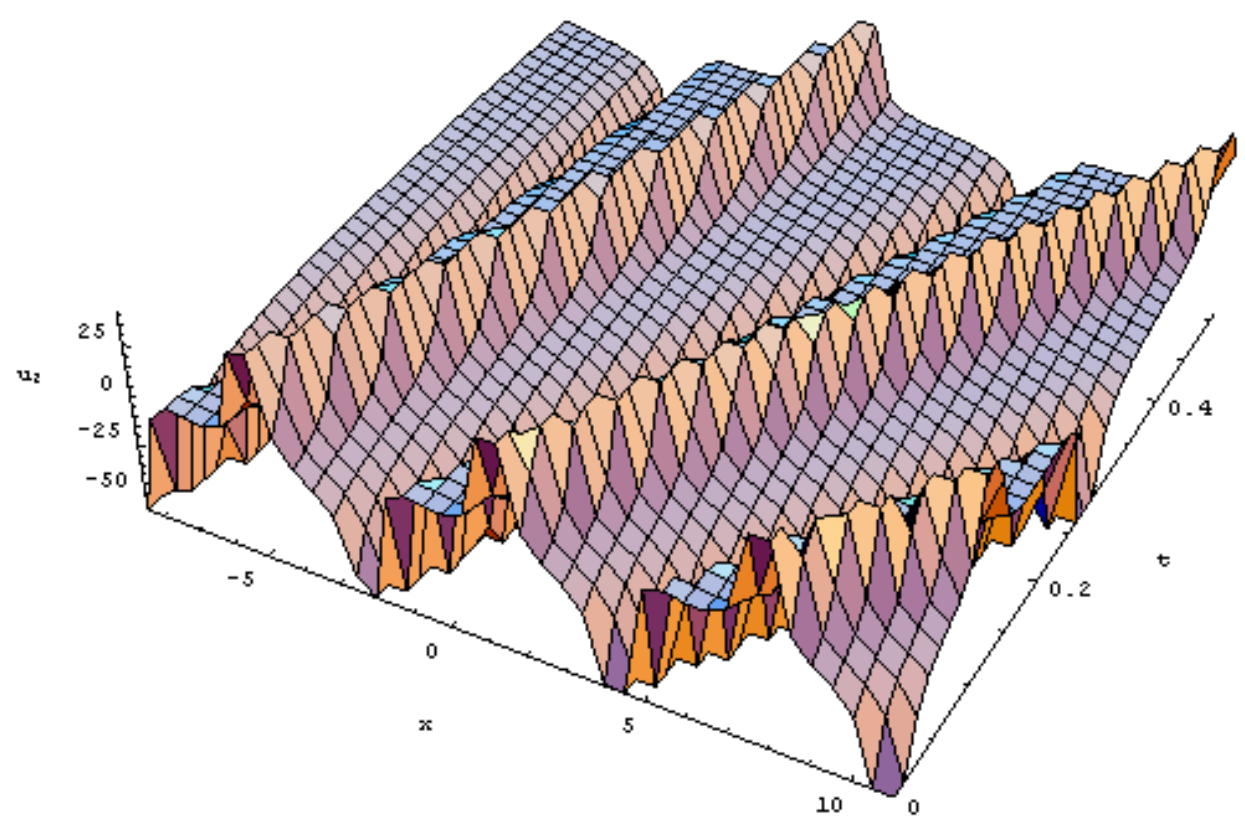

(a)

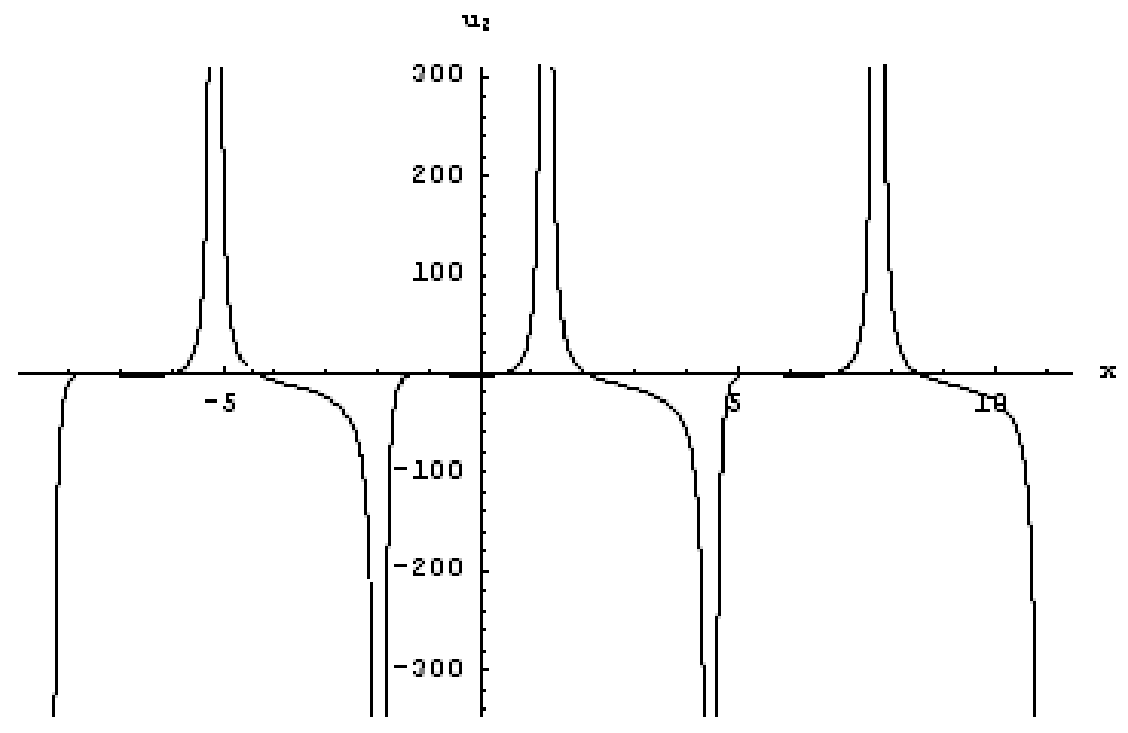

(b)

Figure 1. (a) Simulation of $u_{2}$ when $k_{0}=0, k_{5}=M=\alpha(t)=\beta(t)=\gamma(t)=1, m=0.1$.

(b) Plane graph when $t=0$. 


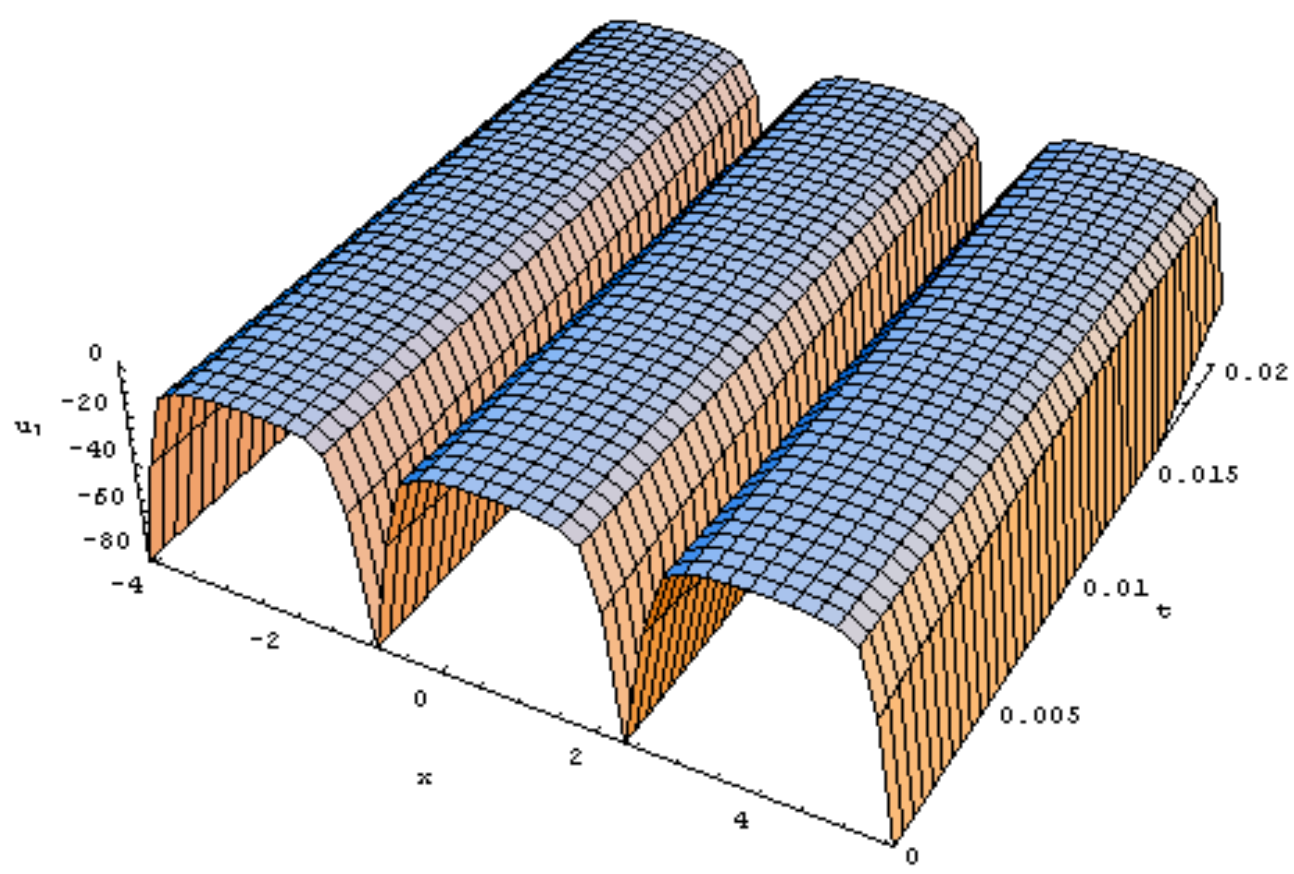

(a)

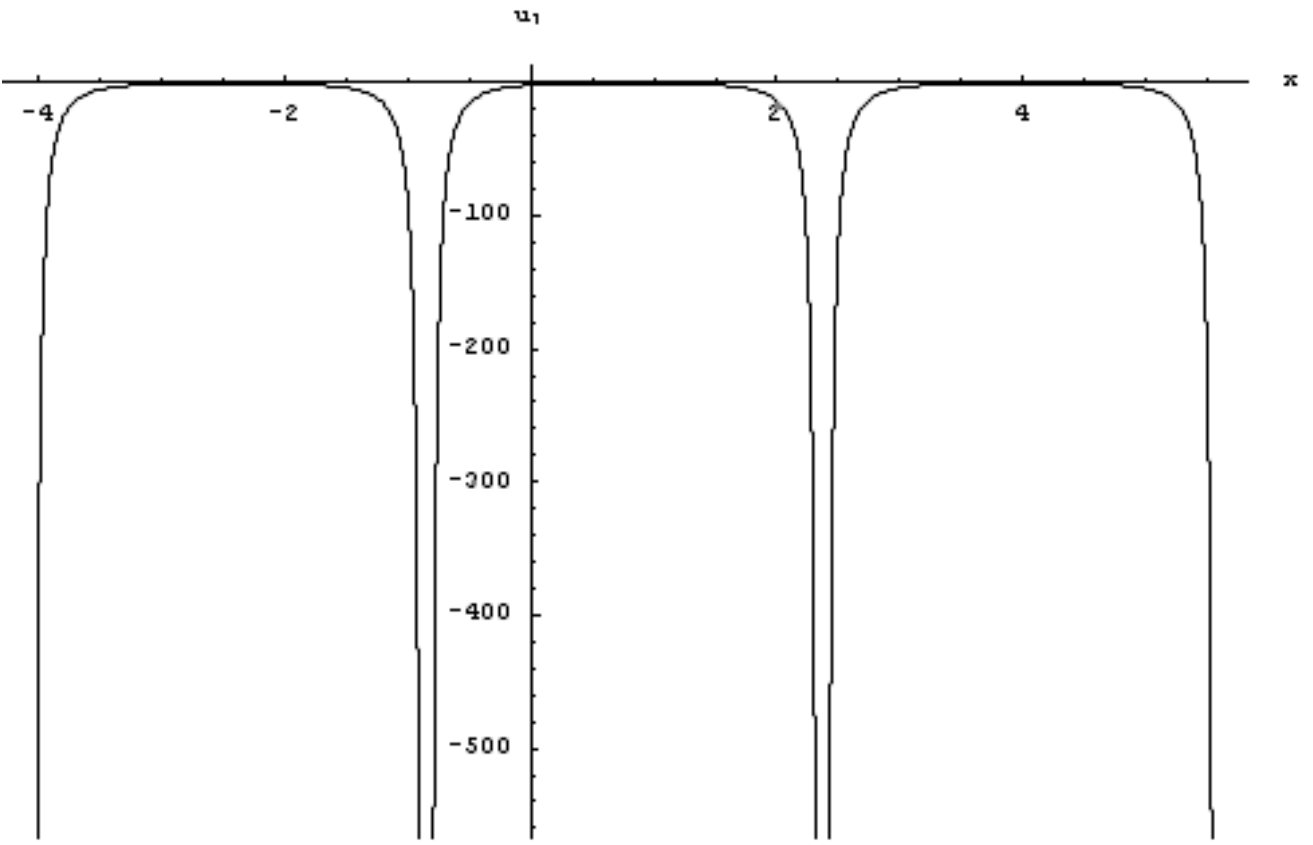

(b)

Figure 2. (a) Simulation of $u_{7}$ when $k_{0}=0, k_{5}=M=\alpha(t)=\beta(t)=\gamma(t)=1, m=0.1$.

(b) Plane graph when $t=0$. 
Remark 5: The eight types of explicit solutions except $u_{4}$ we obtained here to Eq. (8) are not shown in the previous literature to our knowledge. They are new exact solutions of Eq.(8). Solutions $u_{i(i=1,4,7,9)}$ are degenerated to solitary-like solutions when the modulus $m \rightarrow 1$, and solutions $u_{i(i=1,2,3,7,8)}$ are degenerated to triangular functions solutions when the modulus $m \rightarrow 0 . k_{0}$ and $k_{5}$ are arbitrary constants in all above cases.

\section{CONCLUSION}

In this paper, we succeed to propose a general algebraic method for finding new exact solutions of the GVKdV equation (8). More importantly, our method is much simple and powerful to find new solutions to various kinds of nonlinear evolution equations, such as $\mathrm{KdV}$ equation, Boussinesq equation, zakharov equation, etc. we believe that this method should play an important role for finding exact solutions in the mathematical physics.

Acknowledgments- The authors express their sincere thanks to the referees for their careful reading of the manuscript and constructive suggestion. The work is supported by the National Nature Science Foundation of China (Grant No. 61070231), the Outstanding Personnel Program in Six Fields of Jiangsu (Grant No. 2009188), the Graduate Student Innovation Project of Jiangsu Province (Grant No. CXLX13_673) and the Scientific Research Foundation of NanJing Institute of Technology (Grant No. CKJB201218).

\section{REFERENCES}

1. M.J. Ablowitz, P.A. Clarkson, Soliton, Nonlinear Evolution Equations and Inverse Scattering, Cambridge University Press, New York, 1991.

2. H.Y. Wang, X.B. Hu, Gegenhasi, 2D Toda lattice equation with self-consistent sources: Casoratian type solutions, bilinear Bäcklund transformation and Lax pair, Journal of Computational and Applied Mathematics 202, 133-143, 2007.

3. D.C. Lu, B.J. Hong. Bäcklund transformation and n-soliton-like solutions to the combined $\mathrm{KdV}$-Burgers equation with variable coefficients, International Journal of Nonlinear Science 1(2), 3-10,2006.

4. H.C. Hu, X.Y. Tang, S.Y. Lou, Q.P. Liu,Variable separation solutions obtained from Darboux Transformations for the asymmetric Nizhnik-Novikov-Veselov system. Chaos, Solitons and Fractals 22( 2), 327-334, 2004. 
5. J.H. He, Homotopy perturbation method for bifurcation of nonlinear problems. International Journal of Nonlinear Science and Numerical Simulation 6, 207-208, 2005. 6. W.T. Li, H.Q. Zhang, A new generalized compound Riccati equations rational expansion method to construct many new exact complexion solutions of nonlinear evolution equations with symbolic computation. Chaos, Solitons and Fractals 39, 2369-2377, 2009.

7. J.H. He, Some asymptotic methods for strongly nonlinear equations. International Journal of Modern Physics B 20, 1141-1199, 2006.

8. Y. Feng, H.Q. Zhang, A new auxiliary function method for solving the generalized coupled Hirota-Satsuma KdV system, Applied Mathematics and Computation 200, 283-288, 2008.

9. J.Q. Hu, An algebraic method exactly solving two high dimensional nonlinear evolution equations, Chaos Solitons Fractals 23, 391-398, 2005.

10. W.H. Huang, Y.L. Liu, Jacobi elliptic function solutions of the Ablowitz-Ladik discrete nonlinear Schrodinger system, Chaos, Solitons and Fractals 40, 786-792, 2009. 11. H.A. Abdusalam, On an improved complex Tanh-function method, International Journal of Nonlinear Science and Numerical Simulation 6, 99-106, 2005.

12. E.M.E. Zayed, K.A. Gepreel, Some applications of the G'/G-expansion method to non-linear partial differential equations, Applied Mathematics and Computation 212 , $1-13,2009$.

13. M.L. Wang, X.Z. Li, Extended F-expansion method and periodic wave solutions for the generalized Zakharov equations, Physics Letters A 343, 48-54, 2005.

14. B.J. Hong, New Jacobi elliptic functions solutions for the variable-coefficient mKdV equation, Applied Mathematics and Computation 215(8), 2908-2913, 2009.

15. B.J. Hong, New exact Jacobi elliptic functions solutions for the generalized coupled Hirota-Satsuma KdV system, Applied Mathematics and Computation 217 (2), 472-479, 2010.

16. B.J. Hong, D.C. Lu, New Jacobi elliptic function-like solutions for the general KdV equation with variable coefficients, Mathematical and Computer Modelling 55, 1594-1600, 2012.

17. X.Q. Zhao, D.B. Tang, L.M. Wang, New soliton-like solutions for KdV equation with variable coefficient, Physics Letters A 346, 288-291, 2005.

18. J.M. Zhu, C.L. Zheng, Z.Y. Ma, A general mapping approach and new travelling wave solutions to the general variable coefficient $\mathrm{KdV}$ equation, Chinese Physics 13(12), 2008-2012, 2004.

19. Z.T. Fu, S.D. Liu, S.K. Liu, Q. Zhao, New exact solutions to KdV equations with variable coefficients or forcing, Applied Mathematics and Mechanics (English Edition) 25, 73-79, 2004.

20. J.F. Zhang, F.Y. Cheng, Truncated expansion method and new exact solution-like solution of the general variable coefficient KdV equation, Acta Physica Sinica 50(9), 1648-1650, 2001. 
21. W.L. Chan, K.S. Li, Nonpropagating solitons of the variable coefficient and nonisospectral Korteweg-de Vries equation, Journal of Mathematical Physics 30(11), 2521-2526, 1989.

22. T. Chou, Symmetries and a hierarchy of the general KdV equation, Journal of Physics A: Mathematical and General 20, 359-366, 1987.

23. S.Y. Lou, H.Y. Ruan, Conservation laws of the variable coefficient KdV and MKdV equations, Acta Physica Sinica 41(2), 182-187, 1992.

24. W.L. Chan, X. Zhang, Symmetries, conservation laws and Hamiltonian structures of the nonisospectral and variable coefficient $\mathrm{KdV}$ and $\mathrm{MKdV}$ equations, Journal of Physics A: Mathematical and General 28, 407-412, 1995.

25. E.G. Fan, The integrability of nonisospectral and variable-coefficient KdV equation with binary Bell polynomials, Physics Letters A 375(3), 493-497, 2011.

26. J.F. Zhang, P. Han, Symmetries of the Variable Coefficient KdV Equation and Three Hierarchies of the Integro differential Variable Coefficient KdV Equation, Chinese Physics Letters 11(12), 721-723, 1994. 